\title{
Phylogenetic analysis of five medically important Candida species as deduced on the basis of small ribosomal subunit RNA sequences
}

\author{
Lydia Hendriks, ${ }^{1}$ Anne Goris, ${ }^{1}$ Yves Van de Peer,${ }^{1}$ Jean-Marc Neefs, ${ }^{1}$ \\ Marc Vancanneyt, ${ }^{2}$ Karel Kersters, ${ }^{2}$ Gregoire L. Hennebert ${ }^{3}$ and \\ RUPERT DE WACHTER ${ }^{1 *}$ \\ ${ }^{1}$ Departement Biochemie, Universiteit Antwerpen (UIA), Universiteitsplein 1, B-2610 Antwerpen, Belgium \\ ${ }^{2}$ Laboratorium voor Microbiologie, Rijksuniversiteit Gent, Ledeganckstraat 35, B-9000 Gent, Belgium \\ ${ }^{3}$ Mycothèque de l'Université Catholique de Louvain, Place Croix du Sud 3, B-1348 Louvain-la-Neuve, Belgium
}

(Received 21 September 1990; revised 12 December 1990; accepted 7 January 1991)

\begin{abstract}
The classification of species belonging to the genus Candida Berkhout is problematic. Therefore, we have determined the small ribosomal subunit RNA (srRNA) sequences of the type strains of three human pathogenic Candida species; Candida krusei, $C$. lusitaniae and $C$. tropicalis. The srRNA sequences were aligned with published eukaryotic srRNA sequences and evolutionary trees were inferred using a matrix optimization method. An evolutionary tree comprising all available eukaryotic srRNA sequences, including two other pathogenic Candida species, $C$. albicans and $C$. glabrata, showed that the yeasts diverge rather late in the course of eukaryote evolution, namely at the same depth as green plants, ciliates and some smaller taxa. The cluster of the higher fungi consists of 10 ascomycetes and ascomycete-like species with the first branches leading to Neurospora crassa, Pneumocystis carinii, Candida lusitaniae and $C$. krusei, in that order. Next there is a dichotomous divergence leading to a group consisting of Torulaspora delbrueckii, Saccharomyces cerevisiae, $C$. glabrata and Kluyveromyces lactis and a smaller group comprising $C$. tropicalis and $C$. albicans. The divergence pattern obtained on the basis of srRNA sequence data is also compared to various other chemotaxonomic data.
\end{abstract}

\section{Introduction}

Since the classification of higher fungi, Ascomycotina and Basidiomycotina, is based mainly on the morphology of the sexual asco- or basidiospores, other classification criteria have to be used for the Deuteromycotina. The classification of the genus Candida Berkhout, based on the morphology of asexual structures (hyphae, pseudohyphae and sympoduloconidia), as well as various biochemical properties, resulted in an artificial grouping. After the discovery by van der Walt \& Hopsu-Havu (1976) of the cell wall reaction to diazonium blue B, the genus Candida Berkhout as presented by Meyer et al. (1984) was shown to be heterogeneous and composed of ascomycete- as well as basidiomycete-related yeasts. Recently, the genus Candida Berkhout has been redefined, mainly on the basis of cell wall properties

\footnotetext{
Abbreviation: srRNA, small ribosomal subunit RNA
}

The nucleotide sequence data reported in this paper have been submitted to GenBank and have been assigned the accession numbers M55526 (C. lusitaniae), M55527 (C. tropicalis) and M55528 (C. krusei).
(Weijman et al., 1988), and now comprises only yeasts with an ascomycete affiliation. The yeasts with a basidiomycete relationship have been placed in the genera Rhodotorula and Cryptococcus. In spite of this new classification the genus Candida still exhibits some heterogeneity due to the affiliation of some species to different teleomorphic ascomycete genera. A historical review of the classification of the genus Candida is given by Viljoen \& Kock (1989a).

The genus Candida (sensu Weijman et al., 1988) includes several human pathogens. Infections caused by C. albicans and $C$. tropicalis occur most frequently (Ahearn, 1978). In addition, C. krusei, C. lusitaniae and C. glabrata have been described as opportunistic pathogens (Ahearn, 1978; Blinkhorn et al., 1989).

For $C$. albicans, $C$. tropicalis and $C$. glabrata no teleomorphic counterparts have been described. In contrast, $C$. krusei and C. lusitaniae correspond respectively to Issatchenkia orientalis and Clavispora lusitaniae, two ascosporogenous yeasts (Kreger-van Rij, 1984). These anamorph/teleomorph relationships are proven by several chemotaxonomic data (Viljoen et al., 1989, and references cited there). 
The ascosporogenous affiliation for $C$. albicans, $C$. tropicalis, C. glabrata, C. lusitaniae and C. krusei is supported by a negative diazonium blue $B$ staining reaction (Van der Walt \& Hopsu-Havu, 1976; Hagler \& Ahearn, 1981) and a negative urease test (Hagler \& Ahearn, 1981).

Coenzyme $\mathrm{Q}$ analysis (the number of isoprene units per ubiquinone molecule) revealed that $C$. albicans and C. tropicalis are characterized by a Q-9 system. C. lusitaniae and $C$. krusei have a Q-8 and Q-7 system, respectively, while $C$. glabrata is characterized by a Q-6 system (Yamada \& Kondo, 1972; Yamada et al., 1976). Other studies have shown a high similarity between $C$. albicans and $C$. tropicalis, e.g. fatty acids analysis (Viljoen \& Kock, 1989b; Viljoen et al., 1989), proton magnetic resonance studies of mannans (Spencer \& Gorin, 1969), antigenic comparison of enzymes (Bruneau et al., 1985), an analysis based on 79 phenotypic characters (Odds $e t$ $a l ., 1990)$ and protein electrophoresis patterns (Vancanneyt et al., 1991). Furthermore, C. albicans, C. lusitaniae, C. tropicalis and C. krusei are placed in the same group because of the presence of linoleic and linolenic acids in their fatty acid profile. C. glabrata is differentiated from these four species by the absence of linoleic and linolenic acid (Viljoen \& Kock, 1989b).

In order to obtain more information on the evolutionary positions of five pathogenic Candida species and to enlarge our knowledge of the scope of the heterogeneous genus Candida, the small ribosomal subunit RNA (srRNA) gene sequences of the type strains of the species C. tropicalis, C. lusitaniae and C. krusei were determined and aligned with those from other organisms (Neefs et al., 1990) including C. albicans (MUCL 20800, type strain) (Hendriks et al., 1989) and C. glabrata (CBS 138, type strain) (Wong \& Clark-Walker, 1990). As mentioned above, the five Candida species are well characterized for various phenotypic markers, viz. fatty acid profiles, coenzyme $Q$ system and diazonium blue reaction, which enables us to compare our results with analyses based on other criteria. Only the type strain of each Candida species was investigated because the intraspecific homogeneity of these taxa was demonstrated by Vancanneyt et al. (1991). Starting from the alignment of the srRNA sequences, evolutionary trees were constructed using a matrix method.

\section{Methods}

Yeast strains. The Candida strains studied are all type strains and are listed in Table 1 . The yeasts were grown in $2 \%(\mathrm{w} / \mathrm{v})$ agar containing $2 \%(\mathrm{w} / \mathrm{v})$ glucose, $1 \%(\mathrm{w} / \mathrm{v})$ peptone, $0.5 \%(\mathrm{w} / \mathrm{v})$ yeast extract and $3 \%$ $(\mathrm{w} / \mathrm{v}) \mathrm{CaCO}_{3}$ at $28{ }^{\circ} \mathrm{C}$. Cells were collected and washed twice with $0.01 \mathrm{M}$-phosphate $\left(\mathrm{KH}_{2} \mathrm{PO}_{4} / \mathrm{Na}_{2} \mathrm{HPO}_{4}\right)$ buffer $\mathrm{pH} \mathrm{7.0}$ and stored at $-70^{\circ} \mathrm{C}$ before use.
Table 1. Yeast type strains used in this study

\begin{tabular}{|c|c|c|c|}
\hline Yeast & MUCL no.* & CBS no. $\dagger$ & $\begin{array}{l}\text { Restriction enzyme } \\
\text { fragment used } \ddagger\end{array}$ \\
\hline Candida lusitaniae & MUCL $29855 \S$ & CBS $4413 \S$ & PstI $(9.0 \mathrm{~kb})$ \\
\hline Candida krusei & MUCL $29849 \S$ & CBS $573 \S$ & PstI (6.7 kb) \\
\hline Candida tropicalis & MUCL $30002 \|$ & CBS 2310\| & HindIII $(9.0 \mathrm{~kb})$ \\
\hline
\end{tabular}

* MUCL, Mycothèque de l'Université Catholique, Louvain-laNeuve, Belgium.

† Corresponding CBS (Centraalbureau voor Schimmelcultures, Baarn, The Netherlands) number.

$\ddagger$ Restriction fragment containing the srRNA gene which was used for sequencing listed together with its length in basepairs.

$\S$ Type strain.

|| Type strain of Candida vulgaris Berkhout, original name of the type species of the genus and synonym of Candida tropicalis.

Isolation of genomic DNA. High molecular mass DNA ( $>50 \mathrm{~kb}$ ) was isolated by grinding $2 \mathrm{~g}$ of yeast cells in a mortar in the presence of $1 \mathrm{~g}$ alumina and liquid nitrogen. The crushed cells were then suspended in $50 \mathrm{~mm}$-Tris, $10 \mathrm{~mm}-\mathrm{MgCl}_{2}, 50 \mathrm{~mm}-\mathrm{NaCl}, 1 \%$ w/v, SDS, adjusted with $\mathrm{HCl}$ to $\mathrm{pH} 7 \cdot 4$. Phenol extraction and nucleic acids precipitations were done as described in Sambrook et al. (1989).

Cloning and sequencing. Genomic yeast DNA was digested with a set of hexamer-recognizing restriction enzymes. The DNA fragments thus generated were separated on a $0.8 \%(w / v)$ agarose gel $\left(1.2 \mathrm{~V} \mathrm{~cm}^{-1}, 16 \mathrm{~h}\right)$ and blotted (Southern, 1975) on a Hybond $\mathrm{N}$ plus membrane (Amersham). The membrane was hybridized with an srDNA probe (Hendriks et al, 1989) which was radioactively labelled by nicktranslation (Rigby et al., 1977) using an Amersham nick-translation kit. Hybridization was carried out according to the manufacturer's instructions. Subsequent autoradiography allowed us to identify and select restriction enzymes generating DNA fragments that comprise the entire srRNA gene for each species (Table 1), and which were used for the cloning step. For this purpose, the plasmid vector pUC18 (Yanisch-Perron et al., 1985) was digested with a compatible restriction enzyme and ligated (Sambrook et al., 1989) to the genomic yeast restriction enzyme fragments. Escherichia coli DH5-alpha cells (BRL) were transformed (Hanahan, 1983) with the plasmids and grown on a suitable agar broth (Sambrook et al., 1989). In order to detect srRNA gene-containing recombinants a colony hybridization was performed according to Dale \& Greenaway (1984). These recombinant plasmids were amplified and DNA was extracted using the boiling method (Hoimes \& Quigley, 1981) and used as template for the dideoxy sequencing method (Sanger et al., 1977). For this purpose 16 primers complementary to the coding and non-coding strands of evolutionarily conserved areas of the srRNA gene were used as described by Hendriks et al. (1989). The polymerase used was Sequenase (USB, Cleveland, Ohio, USA) and the reaction conditions were as described by the manufacturer.

Sequence alignment and construction of evolutionary trees. The srRNA sequences of the three Candida species were aligned with other complete srRNA sequences (Neefs et al., 1990). The alignment, which comprises the srRNA sequences of 64 eukaryotic species, is obtainable from the authors in computer-readable form.

For the inference of evolutionary trees by distance matrix methods, dissimilarity values must be computed for all pairwise comparisons of aligned nucleotide sequences. Dissimilarity $(D)$ between each pair of 
sequences A and B is defined as (Van de Peer et al., 1990, Hendriks $e t$ al., 1988)

$$
D_{\mathrm{AB}}=-\frac{3}{4} \ln \left[1-\frac{4}{3}\left(\frac{S}{I+S}\right)\right]\left[1-\frac{G}{T}\right]+\frac{G}{T}
$$

where $I$ is the number of identical nucleotides, $S$ is the number of positions showing a substitution and $G$ is the number of gaps in one sequence with respect to the other. $T$ is the sum of $I, S$ and $G$. The first term of the equation accounts for substitutions and comprises a correction factor for multiple mutations per site (Jukes \& Cantor, 1969; Kimura \& Ohta, 1972). The second term accounts for deletions and insertions and comprises no correction. Adjacent gaps are treated as a single gap.

The resulting dissimilarity matrix, which in general is non-additive, serves as the input for an algorithm developed by De Soete (1983), which computes the additive matrix that optimally approximates the input matrix according to a least squares criterion. Next, the program computes the unique tree defined by this additive matrix.

Either all alignment positions or parts of the alignment, corresponding to evolutionarily conserved areas of the molecule, were taken into account to construct evolutionary trees.

\section{Results and Discussion}

Primary structure and secondary structure of the $\operatorname{sr} R \mathrm{AA}$

The sequences of the srRNA of $C$. krusei, $C$. lusitaniae and $C$. tropicalis are shown in the form of an alignment in Fig. 1 . The location of the $5^{\prime}$ - and $3^{\prime}$-termini were derived by comparison with other srRNA sequences.

In Fig. 2 the primary structure of $C$. kruse $i$ srRNA is folded into a general secondary structure model for eukaryotic srRNAs (Neefs et al., 1990). The helix numbering system is according to the latter paper.

\section{Phylogenetic implications}

The alignment (Neefs et al., 1990), which already comprised the srRNA sequences of the fungi Neurospora crassa, Pneumocystis carinii and Saccharomyces cerevisiae, was completed with the srRNA sequences of $C$. albicans (Hendriks et al., 1989), C. glabrata (Wong \& ClarkWalker, 1990), Kluyveromyces lactis (Maleszka \& ClarkWalker, 1990) and Torulaspora delbrueckii (Hendriks et al., 1990), and with the srRNA sequences of the yeasts examined in the present work. Fig. 3 shows an outline of an evolutionary tree inferred by a matrix optimization method (De Soete, 1983) and comprising 53 srRNA sequences of eukaryotes. Of the 12 Tetrahymena species for which the srRNA sequence is known, only $T$. thermophila was included in the analysis, which reduces the number of eukaryotic species from 64 to 53 . The tree is based on a limited part of the total eukaryotic alignment in which the sequence can be aligned unambiguously. These areas are boxed in Fig. 2 and correspond to more conserved sections of the srRNA molecule. In order to root the tree, an archaeobacterial sequence was included. In the eukaryotic part of the tree in Fig. 3, several early branching protist lineages can be discerned, viz. the diplomonad Giardia lamblia, the microsporidian Vairimorpha necatrix, kinetoplastids plus Euglena gracilis, the amoeba Naegleria gruberi and the slime moulds Physarum polycephalum and Dictyostelium discoideum. Next there is a nearly simultaneous branching into several clusters, viz. metazoa, sporozoa, ciliates plus the dinoflagellate Prorocentrum micans, a cluster consisting of two chromophytes and an oomycete, higher fungi and the green plants. The overall picture is very similar to srRNA trees published previously (e.g. Gunderson et al., 1987; Clark \& Cross, 1988; Hendriks et al., 1988; Nairn \& Ferl, 1988; Rausch et al., 1989; Sogin et al., 1989).

Because srRNA molecules occur universally in all organisms, are not subjected to lateral gene transfer and are slowly ticking molecular clocks, they are virtually the only instruments that allow the evolutionary study of a group as complex and diverse as the eukaryotes on a molecular basis. Comparison with analyses based on other molecular clocks or phenotypic markers is rather difficult because such studies are often limited to a subtaxon of the eukaryotes. However, Ragan (1989) has compared the srRNA trees with biochemical pathway data. In some cases the srRNA tree topology is congruent with the pathway data, while in other cases it is not. Also, when a tree constructed on the basis of 137 ultrastructural, developmental and biochemical characters (Lipscomb, 1989) is compared to the srRNA tree, the same major lines of descent can be discerned in both trees but their branching order differs.

As can be seen from the srRNA tree in Fig. 3 the higher fungi (ascomycetes and ascomycete-like yeasts) diverge rather late in the course of eukaryote evolution. They seem to originate at nearly the same depth as green plants, ciliates and the chromophytes/oomycete cluster.

In order to increase the resolution of the evolutionary distances between the yeast species included in the fungal subcluster, a new tree, comprising only the 10 higher fungi, was constructed. This subtree, depicted in Fig. 4, was computed from a dissimilarity matrix including all alignment positions, using the same algorithm as for the construction of the tree in Fig. 3, and taking Homo sapiens as an outgroup species. Within the fungi the deepest branches lead, in order, to $N$. crassa, $P$. carinii, C. lusitaniae and C. krusei. Next a branch consisting of $C$. albicans and $C$. tropicalis splits off. Finally, there is a dichotomous divergence leading to $C$. glabrata plus $K$. lactis on the one hand and to $T$. delbrueckii plus $S$. cerevisiae on the other hand.

A comparison of the tree (Fig. 4) with the type of ubiquinone system present seems to be most valuable 
c.t. UAUCUGGUUGAUCCUGCCAGUAGUCAUAUGCUUGUCUCAAAGAUUAAGCCAUGCAUGUCUAAGUAUAAGCA--UUAUACAGUGAAACUGCGAAUGGCUCA C.k. UAUCUGGUUGAUCCUGCCA GUAGUCAUAUGCUUGUCUCAAAGAUUAAGCCAUGCAUGUCUAAGUAUAAGCA --UUAUACGGUGAAACUGCGAAUGGCUCA C.1. UAUCUGGUUGAUCCUGCCA GUAGUCAUAUGCUUGUCUCAAAGAUUAAGCCAUGCAUGUCUAAGUAUAAGCAAUUUAUACAGUGMACUGCGAAUGGCUCA

C.1. UUAAAUCAGUUAUCGUUUAUUUGAUAGUACCUUGCUAAUUGGCAUACGCCUGGCAAUUCUAGAGCUAAUACAUGCGUCCAAGCCCGACC-UCUGGAAGGG c.k. UUAAAUCAGUUAUCGUUUAUUUGAUAGUUCCGUUCUACAUGG-AUAACCGUGGAAAUUCUAGAGCUAAUACAUGCGUAAAGCCCCGACUUCGGGAG-GGG c.t. UUAAAUCAGUUAUCGUUUAUUUGAUAGUACCUUACUACUUGG-AUAACCGUGGUAAUUCUAGAGCUAAUACAUGCUUAAAAUCCCGACUGUUUGGAGGG

C.1. CUgUaUUUAUUAGAUAAAAMUCAA--CAUCCG-------Uga UGAUUCAUAAUAACUUGUCGAAUCGCAGGGCCUCGAGCCGGCGAUGGUUCAUUCAAA C.k. -UGUAUUUAUUAGAUAAAAAAUCAAUGCCCUCGGGCUUUUGAUGAUUCAUAAUAACUUUUCGAAGCUCAUGGCCUUGCGC-GGAGCUGGUCAUUCAAA c.t. AUGUAUUUAUUAGAUAAAAAUCAAUGUCUUCGGACUCUUUGAUGAUUCAUAAUAACUUUUCGAAUCGCAUGGCCUUGUGCUGGCGAUGGUCAUUCAAA

C.1. UUUCUGCCCUAUCAACUUUCGAUGGUAGGAUAGAGGCCUACCAUGGUUUCAACGGGUAACGGGAAUUAGGUUCGGUUCCGGAGAGGGAGCCUGAGAAA c.k. UUUCUGCCCUAUCAACUUUCGAUGGUAGGAUAGAGGCCUACCAUGGUUUUCACGGGUAACGGGGAAUAGGGUUCGAUUCCGGAGAGGGAGCCUGAGAAA c.t. UUUCUGCCCUAUCAA CUUUCGAUGGUAGGAUAGUGGCCUACCAUGGUUCAACGGGUA CGGGGAUUAGGGUUCGAUUCCGGAGAGGGAGCCUGAGAAA

C.1. CGGCUACCACAUCCAAGGAGGCAGCAGGCGCGCAMAUUACCCAAUCCCGACACGGGGAGGUAGUGACAAUAAAUAACGAUGCAGGGCCCUUUCGGGUCU C.k. CGGCUACCACAUCCAAGGAAGCAGCAGGCGCGCANAUUACCCAAUCCUGACACAGGGAGGUAGUGACAMUAUAUAACGAUACAGGGCC-UUU--GGUCU C. t. CGGCUACOACAUCCAAGGAAGCAGCAGGCGCGCAAAUUACCCAAUCCCGACACGGGGAGGUAGUGACAAUAAAUAAGAUACAGGGCCCUUUCGGGUCU

C.1. UGUAUUUGGAUGAGUACAAUGUAAAUACCUUAACGAGGACAAUUGGAGGGCAAGUCUGGUGCCAGCAGCCGCGGUAMUUCCAGCUCCAMGAGCGUAUA c.k. UGUAAUUGGAUUGAGUACAAUGUAAAUACCUUAACGAGGAACAAUUGGAGGGCAAGUCUGGUGCCAGCAGCCGCGGUAUUUCCAGCUCCANUAGCGUAUA c.t. UGUAAUUGGAUUGAGUACAAUGUAAAUACCUUAACGAGGAACAAUUGGAGGGCAAGUCUGGUGCCAGCAGCCGCGGUAAUUCCAGCUCCAAAAGCGUAUA

C.1. UUAAAGUGUUGCAGUUAAAAGCUCGUAGUUGAACCUUGGAGGCG------CCGUGCCGUC-CGCUUA-GGCGAGCAC-UGGAGGCGGCG-CCUCUUU-C c.k. UUAAAGUUGUUGCAGUUAAAAACUCGUAGUUGAACUUUGGGCCUGGGCGGACGGUCUACC---UAU--GGUMAGCACUGUUGCGGCCGGGUCUUUCCUU c.t. UUAAAGUUGUGCAGUUAAAA GCUCGUAGUUGAACCUUGGGCUUGGUUGGCCGGUCC-AUCUUUCUGAUGCGUACUGGACCCAACCGAG-CCUUUCCUU

C.1. CUCCUCCUCUUAGCAAUAAGAGGAGGACUGUUACUUUGAGUAAAUGAGAGUGUUCAAAGCAGGCGCACCGCUUGAAUCUGUUAGCAUGGAMUAUUAGAU c.k. CUGGCUAGCCCUCG-GGCGAACCAGGACGAUUACUUUGAGGAAAUUAGAGUGUUCAAAGCAGGCCU-UUGCUCGGAUAUAUUAGCAUGGAUAAUAGAAU c.t. CUGGCUAGCCUUUU-GGCGAACCAGGACUUUUACUUUGAAAAAAUUAGAGUGUUCAAAGCAGGCCU-UUGCUCGAAUAUAUUGGAUGGAAUAAUAGAUU

C.1. AGGACCC-AUGGUUCUAUUUUGUUGGUUUCUAGGACCAUCGUAAUGAUUAAUAGGGACGGUCGGGGGCAUCAGUAUUCAGUUGUCAGAGGUGAAUUCUU C.k. AGGACGC-AUGGUUCUAUUUUGUUGGUUUCUAGGACCAUCGUAAUGAUUAAUAGGGACGGUCGGGGGCAUCAGUAUUCAGUCGUCAGAGGUGAAUUCUU c.t. AGGACGUUAUGGUUCUAUUUUGUUGGUUCUAGGACCAUCGUAAUGAUUAAUAGGACGGUCGGGGUAUCAGUAUUCAGUUGUCAGAGGUGAMAUUCUU

C.1. GgAUUUACUGAaGACUNACUACUGCGAAGCAUUUGCCAAGGACGUUUCAUUAAUCAAGACGAAAGUUAGGGgAUCGAAGAUGAUCAGAUACCGUCGU c.k. GGAUUGA CUGAAGACUMACUACUGCGAAGCAUUUGCCAAGGA CGUUUUCAUUAAUCAMGAACGAMAGUUAGGGGAUCGAAGAUGAUCAGAUACCGUCGU c.t. GGAUUUACUGAAUACUAACUACUGCGAAGCAUUUACCAAGGACGUUUCAUUAAUCAAGACGAA GUUAGGGGAUCGAGAUGAUCAGAUACCGUCGU

C.1. AGUCUUAACCAUAMACUAU GCCGACUAGGGAUCGGGCGGCGUUCAUUUAGUGACGCGCUCGGCACCUUACGAGAMUCAMAGUCUUUGGGUUCUGGGGGG c.k. AGUCUUAACCAUAAACUAUGCCGACUAGGGAUCGGGUGGUGCUACUUU---G-CCCACUCGGCACCUUACGAGAAUCAAAGUUUUUGGGUUCUGGGGGG c.t. AGUCUUAACCAUAAACUAUGCCGACUAGGGAUCGGUUGUUGUUCUUUUAUUGACGCAAUCGGCACCUUACGAGAAUCAAAGUCUUUGGGUUCUGGGGG

C.1. AGUAUGGUCGCAAGGCUGAACUUAAAGGAUUUGACGGAAGGGCACCACCAGGAGUGGAGCCUGCGGCUUAAUUUGACUCAACACGGGGMAACUCACCAG c.k. AGUAUGGUCGCAAGGCUGANAUUAAAGGAUUGACGGAGGGCACCACCAGGAGUGGAGCCUGCGGCUUAAUUUGACUCANCACGGGGAACUCACCAG c.t. AGUAUGGUCGCAAGGCUGAACUUAMAGAUUGACGGAAGGCACCACCAGGAGUGGAGCCUGCGGCUUAAUUUGACUCAMCACGGGGAAAUCACCAC

C.1. GUCCAGACACAAUMAGGAUUGACAGAUUGAGAGCUCUUUCUUGAUUUUGUGGGUGGUGUGCAUGGCCGUUCUUAGUGGUGGAGUGAUUUGUCUGCUUA c.k. GUCCAGA CGUAAUAAGGAUUGACAAGUU-AGAGA CUUUCUCUUGAUCUUACGGGUGGUGGUGCAUGGCCGUUUUUAGUCCUUGGAGUGAUUUGUCUGCUUA c.t. GUCCAGACACANUAAGAUUGACAGAUUGAGAGCUCUUUCUUGAUUUUGUGGGUGGUGGUGCAUGGCCGUUCUUAGUUGGUGGAGUGAUUUGUCUGCUUA

C.1. AUUGCGAUAACGAACGAGACCUUAACCU-CUAAUAGCGCCGCUAGCCUUGUGCUGG-CGCGCGCUUCUUAGGGGGACUAUUGACUUGAAUCGAUGGAA c.k. AUUGCGAUAACGGACGAGACCUUAACCUGCUAMAUAGGGCUGCGAGCAUCUGCUCGG--GUGCUCUUCUUAGAGGGACUAUGGGUAUCNAACCCAUGGA c.t. AUUGCGAUAACGAACGAGA CCUUAACCUACUAAUAGUGCUGCUAGCAUUUGCUGGUAUAGUCACUUCUUAGAGGGACUAUCGAUUUCAMGUCGAUGGA

C.1. GUUUGAGGCAMUAACAGGUCUGUGAUGCCCUUAGACGUUCUGGGCCGCACGCGCGCUACACUGACGGAGCCAGCGAG--UUGACCUUGGCCGAGAGGUCU c.k. GUUUGAGGCAMCAACAGGUCUGUGAUGCCCUUAGACGUUCUGGGCCGCACGCGCGCUACACUGACGGAGCCAGCAAGU-CCAACCUUGGUCGAGAGGCCC c.t. GUUUGAGGCAAUACAGGUCUGUGAUGCCCUUAGACGUUCUGGGCCGCACGCGCGCUACACUGACGGAGCCAGCGAGUAUAACCUUGGCCGAGAGGCCU

C.1. GGGMAUCUUGGGMACUCCGUCGUGCUGGGGAUAGAGCAUUGCAMUNUUGCUCUUCAACGAGGAAUUCCUAGUMAGCGCAAGUCAUCAGCUUGCGUUG C.k. GGGUAAUCUCGUGAACUCCGUCGUGCUGGGGAUAAACAUUGUAAUUUUUGGUCUUCAACGAGGAUUUCCUAGUAAGCGCAAGUCAUCAGCUUGCGUUG c.t. GGGAANUCUUGUGAAACUCCGUCGUGCUGGGGAUAIJAGCAUUGUAAUUGUUGCUCUUCAACGAGGAUUUCCUAGUAAGCGCAAGUCAUCAGCUUGCGUUG

C.1. AUUACGUCCCUGCCCUUUGUACACACCGCCCGUCGCUACUACCGAUUGAAUGGCUUAGUGAGGCCUCCGGAUUUGUCUAAGCCGAGG---CA-CCUUGGC c.k. AUUACGUCCCUGCCCUUUGUACACACCGCCCGUCG CUACUACCGAUUGAUUGGCUUAGUGAGGCUUCAMGAUUGGCGCCGCGGGAGGG-GCAACUUUCCC c.t. AUUACGUCCCUGCCCUUUGUACACACCGCCCGUCGCUACUACCGAUUGANUGGCUUAGUGAGGCUUCCGGAUUGG-UUUAGGAMAGGGGGCAA-CUCCAU

C.1. GGUGA--CGGAGNAGCUGGUCANACUUGGUCAUUUAGAGGMGUAMAGUCGUMACAGGUUUCCGUAGGUGMCCUGCGGAAGAUCAUUA c.k. A-UGGGGCCGAGAUCUAGUCAAACUUGGUCAUUUAGAGGUCGUAMAGUCGUAACAGGUUUCCGUAGGUGACCUGCGGAGGAUCAUUA c.t. UCUGGACCGAGAGCUAGUCAAACUUGGUCAUUUAGAGGAGUAAAAGUCGUACAAGGUUUCCGUAGGCGAACCUGCGGAGGAUCAUUA

Fig. 1. Alignment of the srRNA sequences of $C$. tropicalis (C.t.), C. krusei (C.k.) and C. lusitaniae (C.1.). 


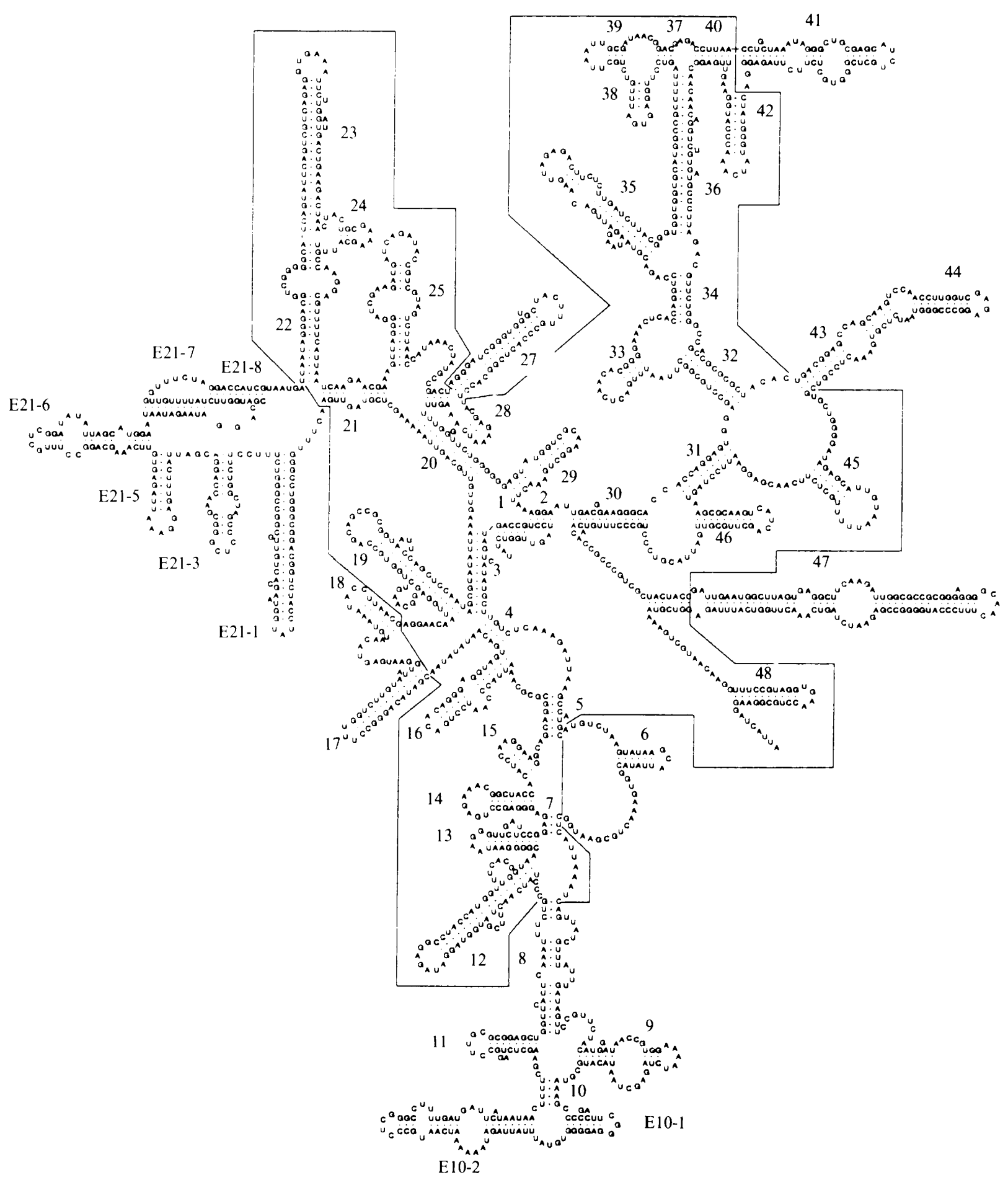

Fig. 2. Secondary structure model of the srRNA of $C$. krusei. The helix numbering in area E21- $n$ is not continuous because helices E21-2 and E21-4, which are found only in the srRNAs of a limited number of species, are missing in $C$. krusei as well as in the two other Candida species, $C$. tropicalis and $C$. lusitaniae. The boxed area indicates more conserved parts of the molecule which can be aligned unambiguously among eukaryotes. The secondary structure models of $C$. tropicalis and $C$. lusitaniae srRNAs are very similar to that of C. krusei. 


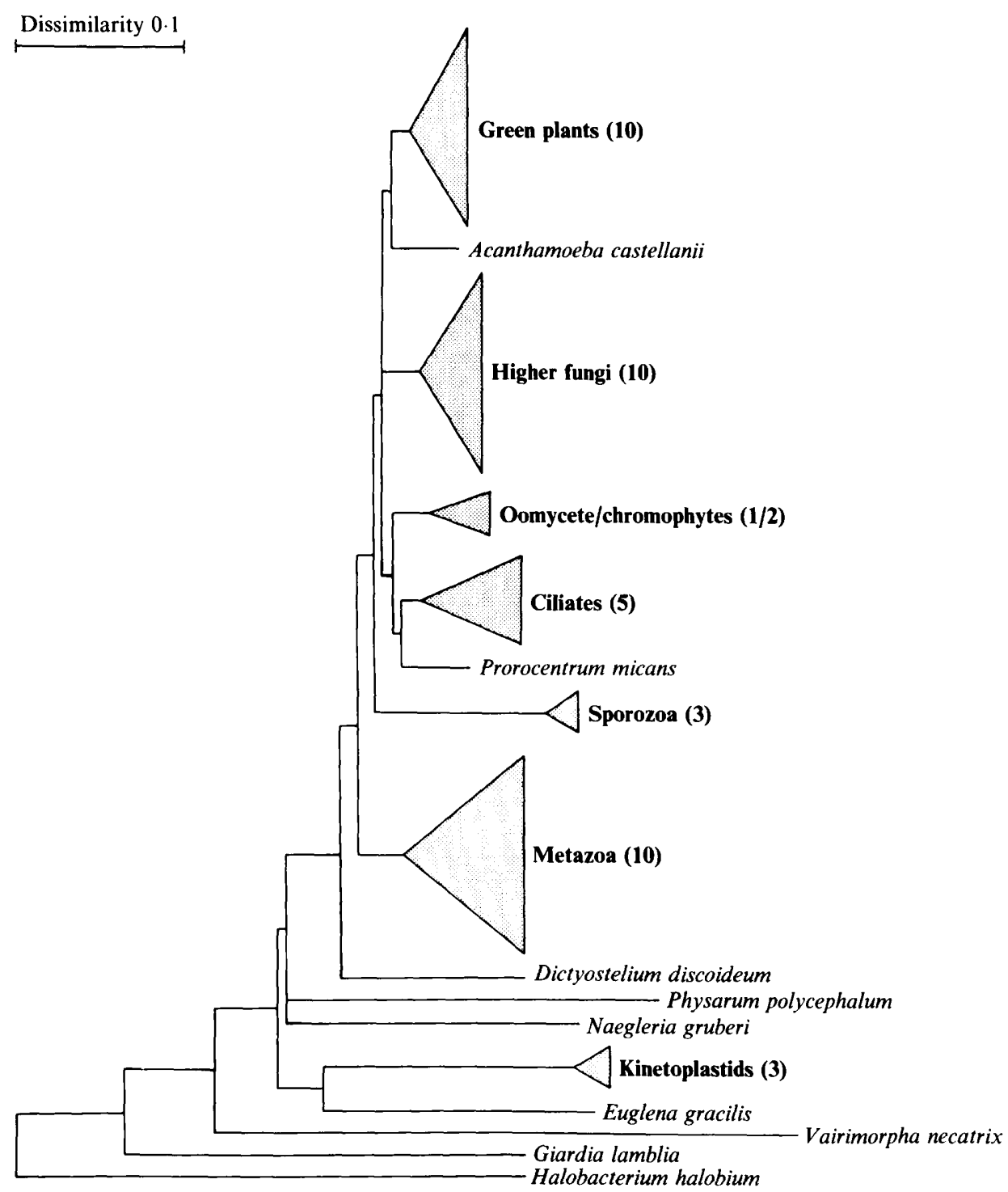

Fig. 3. Simplified version of an evolutionary tree based on 1263 unambiguously aligned positions (comprising the boxed area of Fig. 2) of srRNA sequences of 52 eukaryotes. Clusters are represented as isosceles triangles with a height equal to the mean distance from the deepest branching point to the species forming the cluster, and a base proportional to the number of species. Taxon designations are placed to the right of the corresponding cluster followed by the number of organisms included in the cluster. The dissimilarity between two groups is obtained by summing the lengths of the connecting branches along the horizontal axis, using the scale at the top. Branches originating at levels differing less than $\mathbf{0 . 0 0 2 5}$ in dissimilarity are drawn as diverging simultaneously. The root was placed such as to equalize the distance to the outgroup species (the archaeobacterium Halobacterium halobium) and the average distance to all eukaryotes.

because the coenzyme $Q$ number appears to be conserved in most sporogenous and some asporogenous yeast genera. The type of ubiquinone would thus seem to be a significant generic parameter and ranges between Q-6 and Q-10 among the ascosporogenous genera. Genera with the same number may differ markedly in other properties (Kreger-van Rij, 1984). Fig. 4 shows that four yeast species with a Q-6 system, $T$. delbrueckii, $S$. cerevisiae, C. glabrata and K. lactis (Yamada \& Kondo, 1972; Yamada et al., 1976), group together. Both species possessing the Q-9 system, C. albicans and C. tropicalis, represent a separate cluster. $C$. krusei and $C$. lusitaniae are more distantly related in our tree and are characterized by a Q-7 and Q-8 system, respectively (Yamada \& Kondo, 1972). We conclude that, at least for this subset of yeast species, the srRNA sequences and the ubiquinone system yield congruent results.

We also compared the srRNA sequence similarity with other chemotaxonomic markers which were analysed using alternative treeing methods often based 
Dissimilarity $0 \cdot 1$

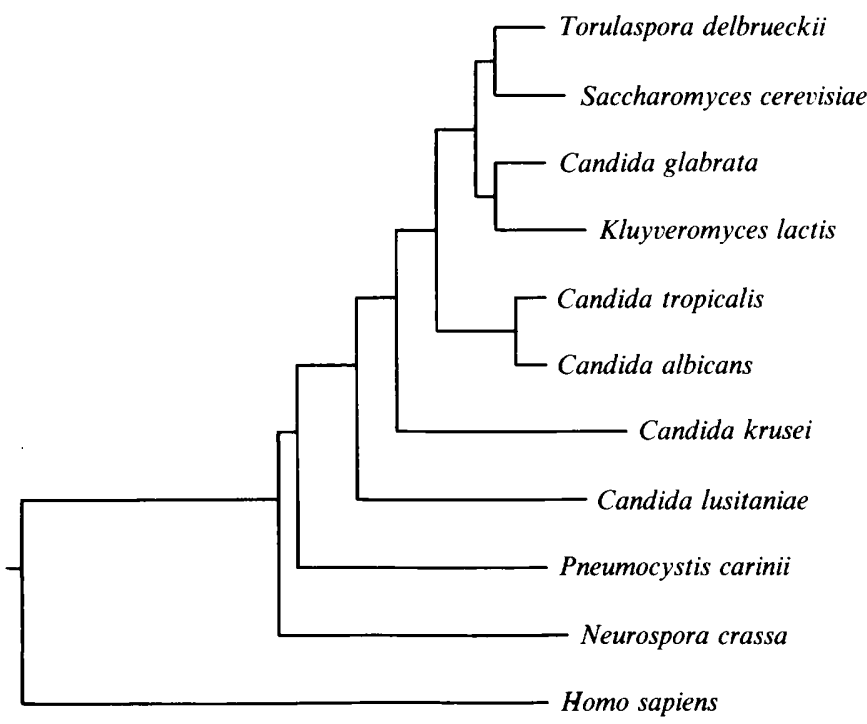

Fig. 4. Evolutionary tree comprising 10 fungal species. All alignment positions are used to construct the tree by a matrix optimization method. Placement of the root was as in Fig. 3, using Homo sapiens as the outgroup species.

on different subsets of species. The close relationship between C. albicans and C. tropicalis (Fig. 4) is also seen on the basis of phenotypic and biochemical criteria (Spencer \& Gorin, 1969; Bruneau et al., 1985; Viljoen \& Kock, 1989a,b; Odds et al., 1990; Viljoen et al., 1989; Vancanneyt et al., 1991). Furthermore, a close evolutionary relationship between $S$. cerevisiae and $C$. glabrata, as shown in Fig. 4, is confirmed by a combined analysis of long-chain fatty acid profiles, cell differentiation and carbon source utilization (Viljoen et al., 1988). In the tree in Fig. 4, S. cerevisiae and T. delbrueckii seem to be very closely related. However, on the basis of electrophoretic whole-cell protein patterns (Vancanneyt et al., 1991) and $\mathrm{mol} \% \mathrm{G}+\mathrm{C}$ values (Kreger-van Rij, 1984) the latter relationship is not detected. Based on protein electrophoresis patterns (Vancanneyt et al., 1991), the divergence order of $C$. albicans, $C$. tropicalis, $C$. krusei and $C$. lusitaniae is analogous to that depicted in the tree in Fig. 4. In contrast, a combined analysis of fatty acid profiles and electrophoretic karyotype yielded other results with C. albicans, $C$. tropicalis and C. lusitaniae forming part of the same subgroup while $C$. krusei belongs to another subgroup (Viljoen \& Kock, 1989b).

In conclusion, inconsistencies can be detected when one compares evolutionary trees based on different chemotaxonomic criteria and on srRNA sequence analysis. These discrepancies can be due to the fact that different sets of organisms and/or other treeing algorithms have been used. Alternatively, the discrepancies between the trees can also be a consequence of the fact that some of the chemotaxonomic markers used may not be suitable for a study of the relationships among species which are not very closely related. Fatty acid profiles, electrophoretic karyotype analysis and protein electrophoresis profiles have proven their usefulness for the determination of the teleomorph/anamorph relationship between species and/or for the inference of the relationship between species (or strains) which are evolutionarily close, as is the case for $C$. albicans and C. tropicalis. However, the type of ubiquinone system and the srRNA analysis may be more suitable for the study of less related organisms.

Analysis of srRNA sequences has already been successfully applied to studies on the evolution of bacteria (Woese, 1987) and protists (Gunderson et al., 1987; Sogin et al., 1989). The results presented in this work, though still limited, also show the potential of srRNA analysis for the deduction of evolutionary relationships among yeast species. To define the status of the majority of Candida species, many more sequences of ascomycetes and ascomycete-like yeasts should be determined.

This research was supported by grants Bio/03, Bio/14 and Bio/11 of the Belgian National Incentive Programme on Fundamental Research in Life Sciences initiated by the Office of Science Policy Programming, and by the FGWO. Yves Van de Peer and Jean-Marc Neefs hold an IWONL scholarship.

\section{References}

AHEARN, D. G . (1978). Medically important yeasts. Annual Reviews of Microbiology 32, 59-68.

Blinkhorn, R. J., Adelstein, D. \& Spagnuolo, P. G. (1989). Emergence of a new pathogen, Candida lusitaniae. Journal of Clinical Microbiology 27, 236-240.

Bruneau, S. M., Guinet, R. M. F. \& Sabbagh, I. (1985). Identification and antigenic comparison of enzymes in the genus Candida by means of quantitative immuno electrophoretic methods: taxonomic significance. Systematic and Applied Microbiology 6, 210220.

Clark, C. G. \& Cross, G. A. M. (1988). Small ribosomal RNA sequences from Naegleria gruberi support the polyphyletic origin of amoebas. Journal of Molecular Evolution 5, 512-518.

DaLE, J. W. \& GreenawaY, P. J. (1984). Identification of recombinant plasmid by in situ colony hybridization. In Methods in Molecular Biology, vol. 2, pp. 277-283. Edited by J. M. Walker. Clifton, New Jersey: Humana Press.

DE SoETE, G. (1983). A least squares algorithm fitting additive trees to proximity data. Psychometrika 48, 621-626.

Gunderson, J. H., Elwood, H., Ingold, A., Kindl, K. \& Sogin, M L. (1987). Phylogenetic relationships between chlorophytes, chrysophytes, oomycetes. Proceedings of the National Academy of Sciences of the United States of America 84, 5823-5827.

HaGler, A. N. \& AhEARN, D. G. (1981). Rapid diazonium blue B test to detect basidiomycetous yeasts. International Journal of Systematic Bacteriology 31, 204-208. 
HaNAHAN, D. (1983). Studies on transformation of Escherichia coli with plasmids. Journal of Molecular Biology 166, 557-580.

Hendriks, L., Van BRoeckHoven, C., Vandenberghe, A., VAN DE Peer, Y. \& De WAChter, R. (1988). Primary and secondary structure of the bird spider Eurypelma californica and evolutionary relationships among eukaryotic phyla. European Journal of Biochemistry 177, 15-20.

Hendriks, L., Goris, A., Neefs, J., Van de Peer, Y., Hennebert G. \& DE WACHTER, R. (1989). The nucleotide sequence of the small ribosomal RNA of the yeast Candida albicans and the evolutionary position of fungi among eukaryotes. Systematic and Applied Microbiology 12, 223-229.

Hendriks, L., Goris, A., De BruYn, K., De Wachter, R. (1990). The small ribosomal subunit RNA sequence of the yeast Torulaspora delbrueckii. Nucleic Acids Research 18, 4611

Holmes, D. S. \& Quigley, M. (1981). A rapid boiling method for the preparation of bacterial plasmids. Analytical Biochemistry 114, 193197.

JUKES, T. H. \& CANTOR, C. R. (1969). Evolution of protein molecules. In Mammalian Protein Metabolism, vol. 3, pp. 21-132. Edited by H. N. Munro. New York: Academic Press.

KimURA, M. \& OHTA, T. (1972). On the stochastic model for estimation of mutational distance between homologous proteins. Journal of Molecular Evolution 2, 87-90.

Kreger-VAn RiJ, N. J. W. (1984). In The Yeasts, a Taxonomic Study, pp. 35-42. Edited by N. J. W. Kreger-van Rij. Amsterdam: Elsevier.

LIPSCOMB, D. L. (1989). Relationships among the eukaryotes. In The Hierarchy of Life, pp. 161-178. Edited by B. Fernholm, K. Bremer \& H. Jornvall. Amsterdam, New York \& Oxford: Excerpta Medica.

MaleszKa, R. \& Clark-Walker, G. D. (1990). Sequence of the gene for the cytoplasmic ribosomal RNA small subunit from Kluyveromyces lactis. Nucleic Acids Research 18, 1889.

Meyer, S. A., Ahearn, D. G. \& Yarrow, D. (1984). Candida Berkhout. In The Yeasts, a Taxonomic Study, pp. 585-844. Edited by N. J. W. Kreger-van Rij. Amsterdam: Elsevier.

NAIRN, C. J. \& FerL, R. J. (1988). The complete nucleotide sequence of the small subunit ribosomal RNA coding region for the cycad Zamia pumila: phylogenetic implications. Journal of Molecular Evolution 27, 133-141.

Neefs, J., Van De Peer, Y., Hendriks, L. \& De Wachter, R. (1990). Compilation of small ribosomal subunit RNA sequences. Nucleic Acids Research 18 (suppl.), 2237-2317.

OdDS, F. C., SACKIN, M. J. \& Jones, D. (1990). Numerical taxonomic analysis of imperfect yeast species in Candida and Torulopsis shows no basis for generic separation. Journal of General Microbiology 136, 761-765.

RAGAN, M. A. (1989). Biochemical pathways and the phylogeny of the eukaryotes. In The Hierarchy of Life, pp. 145-160. Edited by B. Fernholm, K. Bremer \& H. Jornvall, Amsterdam, New York \& Oxford: Excerpta Medica.

Rausch, H., Larsen, N. \& Smith, R. (1989). Phylogenetic relationships of the green alga Volvox carteri deduced from small ribosomal subunit RNA comparisons. Journal of Molecular Evolution 29, 255265

Rigby, P. W. J., Dieckmann, M., Rhodes, C. \& Berg, P. (1977). Labelling deoxyribonucleic acid to high specific activity in vitro by nick translation with DNA polymerase I. Journal of Molecular Biology 133, 237-251.

SambrooK, J., Fritsch, E. F. \& Maniatis, T. (1989). Molecular Cloning: a Laboratory Manual. Cold Spring Harbor, NY: Cold Spring Harbor Laboratory.
Sanger, F., Nicklen, S. \& Coulson, A. R. (1977). DNA sequencing with chain-terminating inhibitors. Proceedings of the National Academy of Sciences of the United States of America 74, 5463-5467.

Sogin, M. L., Gunderson, J. H., Elwood, H. J., Alonso, A. R. \& Peattie, D. A. (1989). Phylogenetic meaning of the kingdom concept : an unusual ribosomal RNA from Giardia lamblia. Science 243, 75-77.

SOUTHERN, E. M. (1975). Detection of specific sequences among DNA fragments separated by gel electrophoresis. Journal of Molecular Biology 98, 503-517.

SPENCER, J. F. T \& Gorin, P. A. J. (1969). Systematics of the genus Candida Berkhout: proton magnetic resonance spectra of the mannans and mannose containing polysaccharides as an aid in classification. Antonie van Leeuwenhoek 35, 33-44.

Vancanneyt, M., Pot, B., Hennebert, G. \& Kersters, K. (1991). Differentiation of yeast species based on electrophoretic whole-cell protein patterns. Systematic and Applied Microbiology (in the Press).

VAN De Peer, Y. NeEFs, J. \& De W AChTER, R. (1990). Small ribosomal subunit RNA sequences, evolutionary relationships among different life forms, and mitochondrial origins. Journal of Molecular Evolution 30, 463-476.

VILJOEN, B. C. \& KocK, L. F. (1989a). The genus Candida Berkhout nom conserv. A historical account of its delimitation. Systematic and Applied Microbiology 12, 183-190.

myces and Saccharomyces species. Systematic and Applied Microbiology 10, 116-120.

Viljoen, B. C. \& Kock, L. F. (1989a). The genus Candida Berkhout nom conserv. A historical account of its delimitation. Systematic and Applied Microbiology 12, 183-190.

ViLJOEN, B. C. \& KOCK, L. F. (1989b). A taxonomic study of the yeast genus Candida Berkhout. Systematic and Applied Microbiology 12, 91-102.

Viljoen, B. C., Kock, J. L. F. \& Thoupou, K. (1989). The significance of cellular long-chain fatty acids compositions and other criteria in the study of the relationship between sporogeneous ascomycete species and asporogeneous Candida species. Systematic and Applied Microbiology 12, 80-90.

VAN DER WALT, J. P. \& Hopsu-Havu, V. K. (1976). A colour reaction for the differentiation of ascomycetous and hemibasidiomycetous yeasts. Antonie van Leeuwenhoek 42, 157-163.

Weijman, A. C. M., Rhodriques de Miranda, L. \& van Der Walt, J. P. (1988). Redefinition of Candida Berkhout and the consequent emendation of Cryptococcus Kutzing and Rhodotorula Harrison. Antonie van Leeuwenhoek 54, 545-553.

WoEse, C. R. (1987). Bacterial evolution. Microbiological Reviews 51, $221-271$

Wong, O. C. \& Clark-Walker, G. D. (1990). Sequence of the gene for the cytoplasmic ribosomal RNA small subunit from Candida (Torulopsis) glabrata. Nucleic Acids Research 18, 1888.

YAMADA, Y.\& KoNDO, K. (1972). Taxonomic significance of coenzyme $\mathrm{Q}$ system in yeasts and yeast-like fungi. In Yeasts, Models in Science and Technics, pp. 363-375. Edited by A. Kockova-Kratochovilova \& B. Minarek. Bratislava: Slovak Academy of Science.

Yamada, Y., NojiRI, M., Matsuyama, M. \& Kondo, K. (1976). Coenzyme $\mathrm{Q}$ system in the classification of the ascosporogenous yeast genera Debaryomyces, Saccharomyces, Kluyveromyces, and Endomycopsis. Journal of General and Applied Microbiology 22, 325337.

Yanisch-Perron, C., Vieira, J. \& Messing, J. (1985). Improved M13 phage cloning vectors and host strains: nucleotide sequences of the M13mpl8 and PUC19 vectors. Gene 33, 103-119. 